\title{
Comparative Study on Two Methods for Bite Mark Analysis
}

\author{
Nima A. Osman, Aya Z. Omer, Amal H. Abuaffan* \\ Faculty of Dentistry, University of Khartoum, Sudan
}

*Corresponding Author: Amal H. Abuaffan, Head department of Orthodontic, Paedodontic and Preventive Dentistry, University of Khartoum, Faculty of Dentistry, Sudan, E-mail: Amalabuaffan@yahoo.com

\begin{abstract}
Objectives: The aims of this study are to evaluate the accuracy of two methods for bite mark analysis (overlay and docking techniques) on three different food materials; apple, eggplant and chocolate, to compare the accuracy of bite mark analysis in the three food materials and to identify the most accurate bite mark.
\end{abstract}

Materials and Methods: The participants in this study were instructed to bite on three different food materials; apple, eggplant and chocolate. Two impressions were taken, one from the bite marks by silicone impression material and the second one from the participants' dentitions by alginate impression material. Dental casts were obtained by poured the dental stone in the impressions taken.

Results: The Kruskal-Wallis ANOVA test was used to evaluate the accuracy of the bite mark analysis on the food materials and by Spearman's correlation to compare between the accuracy of the two techniques (overlay technique and docking technique).

Conclusion: The two applied techniques are similar in accuracy for bite mark analysis. The three use food material is reliable for the bite mark analysis with higher reproducibility on the chocolate than on apple and eggplant.

Keywords: bite mark, chocolate, apple, eggplant

\section{INTRODUCTION}

Worldwide the most frequent forms of human traumas is bite inflicted wounds; humans are commonly use teeth as tools and weapons since old times, usually seen in trials of sexual assault, murder, child abuse and also in cases of homicide and suicide. $(1,2)$

Lacerations, abrasions contusions/bruises, petechiae, indentations, erythema and punctures are the common injuries observed with the bite marks, the human facial bite like are frequently observe on the ears, nose and lips are predominant than that of the upper boundary. In addition it detects in the skin, foodstuffs, or other materials found at the crime scene. $(3,4)$

In criminal and civil court cases Bite marks have been regularly used as confirmation for more than half a century. (5) It mostly hand out as a very influential tool and evidence stems from the verity that the details of human denture are virtually inimitable to an individual, even able to discriminate between identical twins because the teeth can be exaggerated by inheritance and highly personal life manner. Stipulation of evidence from early bite mark analysis was not particularly concerned with the genuine performance of the analysis i.e. the probability of correct or false identification. In most serious crimes especially in the late presenting, victims bite marks may be the only physical evidence accessible, those crimes are serious in nature; recording, documenting and describing the injuries carefully is very important. The typical record features are; simple malocclusions; rotation of teeth, displacement of teeth, spacing, and shape of incision edges. (6)

Subsequent to confirm the injury as a bite mark, the comparison with the suspect's teeth or cast model can be performed through variety of methods like image perception technique (7), the xerographic computer-assisted overlay generation, animated-superimposition methods, manual docking and other techniques. (8)

This study used the computer assisted overlay and the manual docking techniques. So when 
the substantial idea is that an image of the incisal edges of the suspect's teeth placed over the scaled 1:1 image of the bite mark, and then it can be observed if it matches each other or not; the method is called overlay. $(9,10)$

The other type of comparison used in case of bite marks in inanimate objects like chocolate, apples etc. is the docking technique, where the dental model of the suspect applied to the cast of the bitten object to determine if they dock or match. (10)

Bite mark an imperative tool in forensic identification, numerous analysis methods have been modified among practitioners depending on different categorical aspects. Accordingly, the evaluation of methods of bite mark analysis is increasingly needed to match the requirements counting cost and material availability according to the practitioner's position in all aspects.

The results of the current study anticipate to serve in investigations of the bite mark evidence reliability and feasibility in Sudan as a developing country through evaluation of accuracy of two simple methods of bite mark analysis thus ease the forensic identification and increase its qualification in criminal trials.

\section{MATERIALS AND METHODS}

In the present study fifteen volunteers dental students, in the Faculty of Dentistry, University of Khartoum, Sudan were examined extra orally and intra orally. An informed consent was obtained from all participants, dental impressions were taken with alginate impression material and then dental cast of the impressions with dental stone were constructed in dental laboratory at the faculty of dentistry (figure1).

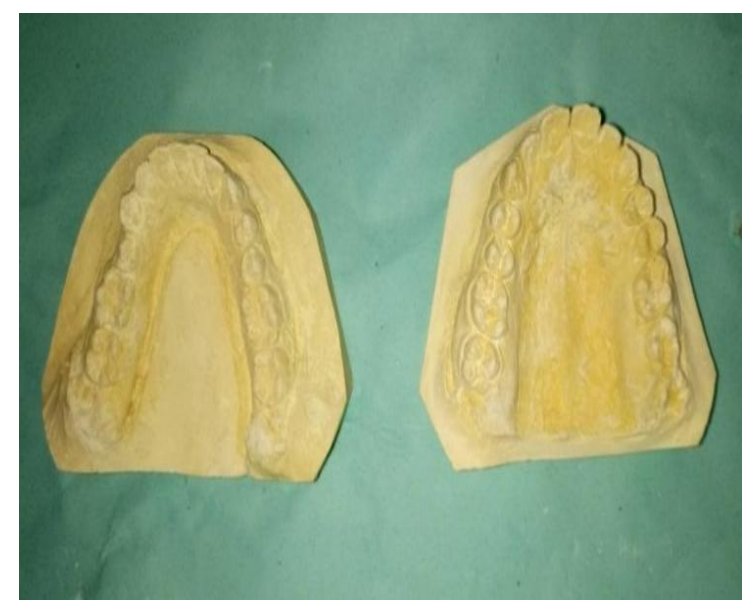

Figure1. Shows die stone cast model for the maxillary and the mandibular jaws of sample 13
The participants were asked to bite in the food materials; apple, eggplant, and chocolate. An impression was taken of the bite marks using light body vinyl polysiloxane, which was applied without pressure; in order to preserve the integrity of the bite mark, it subsequently picked up with a heavy body silicone (figure2).

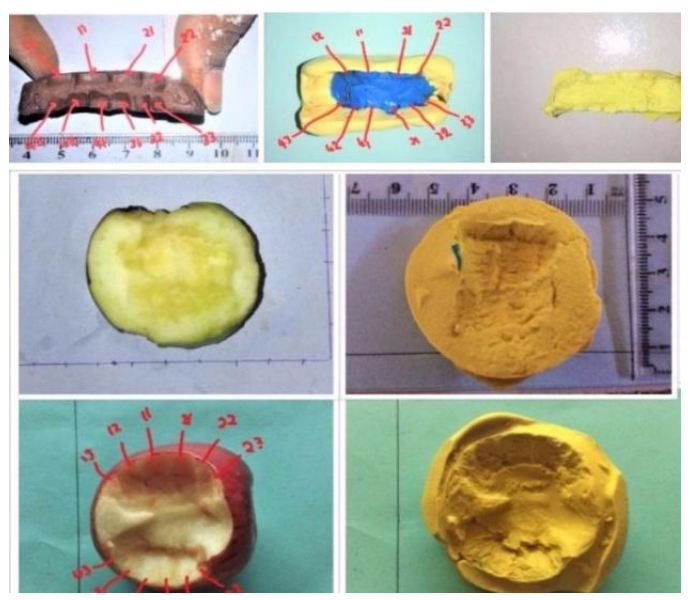

Figure2. Shows the methodology to obtain positive replica of biting surfaces on different food materials (chocolate, eggplant and apple).

The next stage was to pour models from the bite mark impressions with dental stone. The handling of all materials was done according to the manufacturer's instructions. Additionally, photos of the all bite marks and the casts were taken.

In the manual docking (direct) technique, the dental casts of each individual were docked to the die stone cast (positive replica) of the bite mark on apple, eggplant and chocolate to check for matching of incisal edges of the anterior teeth with the bite mark pattern on the food material. Whilst performing the docking technique for apples, eggplants and chocolates with mandibular casts, the positive replicas were reversed to check for matching. The scoring was assigned as

$0=$ not matching (no teeth edge match the impression).

$1=$ slight matching (one or teeth match the impression).

$2=$ moderate (probable) matching (three to four teeth match the impression).

$3=$ excellent (distinctive) matching respectively (five to six teeth match the impression).

The highest score was assigned to the correct match as per modified version of the American Board of Forensic Odontology (ABFO) scoring system for bite marks (figure3). 


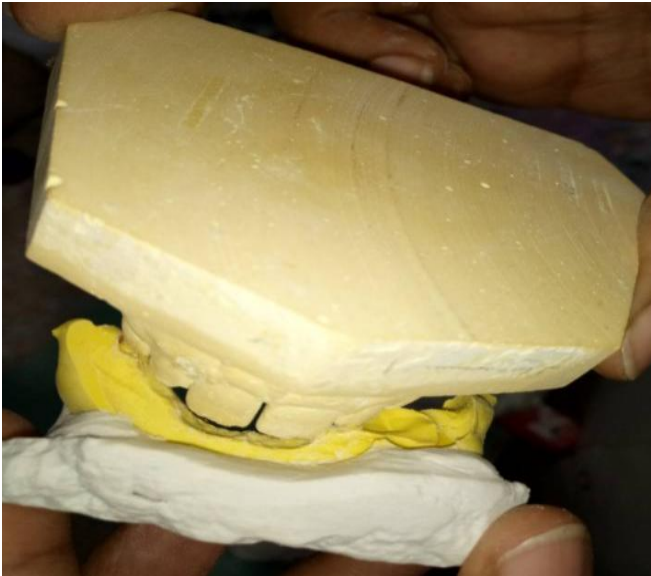

Figure3. Shows the manual docking technique on the cast model of chocolate bite mark using Maxillary cast of sample 13

In the overlay generation (indirect) technique images of one dental cast were imported into a software program (Adobe Photoshop 7.0.1). In the software, the biting surfaces of the maxillary incisor teeth were traced semi automatically (using the 'Magic Wand' tool, at a tolerance level ranging between 4 and 12). The conversion of the image to Life Size enabled a 1:1 (Life Size) superimposition (on the computer) of the biting surfaces and its comparison with the bite mark

Superimposition of the traced biting surfaces onto the bite mark on image .The scoring was then assigned as:

$0=$ for not matching (no incisal edge matches the image).

$1=$ for slight (consistent) matching (one to two teeth matches the image).

$2=$ for moderate (probable) matching (three to four teeth matches the image).

$3=$ for excellent (distinctive) matching respectively (five to six teeth matches the image ). The highest score was assigned to the correct match

Table1. Kruskal-Wallis ANOVA test for reliability of bite mark analysis on food material by Manual docking method

\begin{tabular}{|l|l|l|l|}
\hline Food material & Frequency & Percentage & P-value \\
\hline APPLE & & & \\
No matching & 0 & 00.00 & \\
Slight matching & 6 & 40.00 & \\
Moderate matching & 7 & 46.67 & \\
Excellent matching & 2 & 13.33 & 0.392 \\
Total & 15 & 100.00 & \\
\hline EGGPLANT & & & \\
No matching & 0 & 00.00 & \\
Slight matching & 5 & 33.33 & \\
Moderate matching & 6 & 40.00 & \\
Excellent matching & 4 & 26.67 & \\
Total & 15 & 100.00 & \\
\hline
\end{tabular}
system for bite marks (figure4).

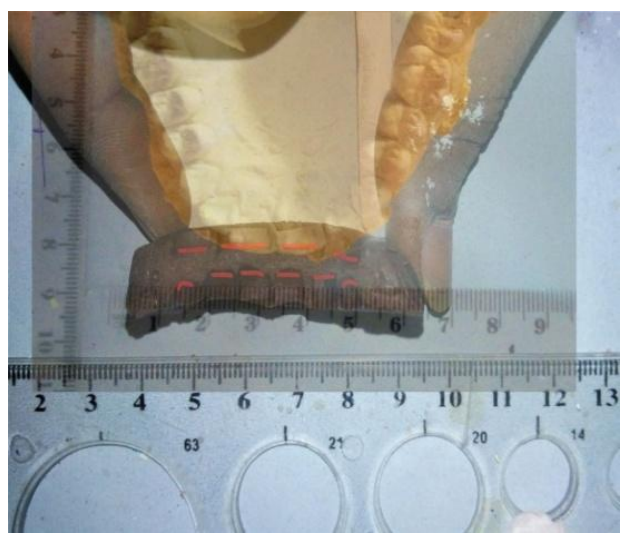

Figure4. Shows the computer assisted overlay generation by Adobe Photoshop software from Photograph of dental cast and photographs of bite mark on chocolate

\section{RESULTS}

The results of this study were tabulated and the reliability of both manual docking technique and computer assisted overlay technique for bite mark analysis on the three food stuff was analyzed by Kruskal-Wallis ANOVA test. By manual docking analysis, excellent matching was observed in $13.3 \%$ of the apple samples, $26.6 \%$ of the eggplant samples and $60 \%$ for the chocolate samples Table1.

By computer assisted overlay generation technique, the percentages of excellent matching of bite mark analysis were observed in $40 \%$ of the apple samples, $6.6 \%$ of the eggplant samples and $66.6 \%$ of the chocolate samples Table 2.

The computer assisted overlay generation technique was compared with the manual docking technique by Spearman's correlation, and the Rho coefficient was 0.400 for the apple, 1.000 for the eggplant and 1.000 for the chocolate Table 3. as per modified version of the ABFO scoring 


\begin{tabular}{|l|l|l|l|}
\hline CHOCOLATE & & \\
No matching & 0 & 0 & \\
Slight matching & 2 & 13.33 \\
Moderate matching & 4 & 26.67 \\
Excellent matching & 9 & 60.00 \\
Total & 15 & 100.00 & \\
\hline
\end{tabular}

$P$-value $\leq 0.05$ significant

Table2. Kruskal-Wallis ANOVA test for reliability of bite mark analysis on food materials by Computer assisted overlay generation method

\begin{tabular}{|l|l|l|l|}
\hline Food materials & Frequency & Percentage & P-value \\
\hline APPLE & & 00.00 & 0.392 \\
No matching & 0 & 26.67 & \\
Slight matching & 5 & 33.33 & \\
Moderate matching & 6 & 40.00 & \\
Excellent matching & 15 & 100.00 & \\
Total & & & \\
\hline EGGPLANT & 0 & 00.00 & \\
No matching & 3 & 20.00 & \\
Slight matching & 11 & 73.33 & \\
Moderate matching & 1 & 06.66 & \\
Excellent matching & 15 & 100.00 & \\
Total & 0 & 0 & \\
\hline CHOCOLATE & 1 & 06.66 & \\
No matching & 4 & 26.67 & \\
Slight matching & 10 & 66.66 & \\
Moderate matching & 15 & 100.00 & \\
Excellent matching & & \\
Total & &
\end{tabular}

$P$-value $\leq 0.05$ significant

Table3. Spearman's correlation between two techniques; the manual docking technique and the computer assisted overlay generation technique for the apple, eggplant and chocolate.

\begin{tabular}{|l|l|l|}
\hline Food materials & $\begin{array}{l}\text { Manual docking analysis versus computer } \\
\text { assisted overlay generation technique }\end{array}$ & P-value \\
\hline APPLE & Spearman's Rho correlation coefficient & 0.400 \\
& Significance (two-tailed) & 0.000 \\
EGGPLANT & Spearman's Rho correlation coefficient & 1.000 \\
CHOCOLATE & Significance (two-tailed) & 0.000 \\
& Spearman's Rho correlation coefficient & 1.000 \\
& Significance (two-tailed) & 0.000 \\
\hline
\end{tabular}

$P$-value $\leq 0.05$ significant

\section{DISCUSSION}

Bite mark meant to be a reliable tool in forensic identification of individuals due to the uniqueness characteristics of human dentition. However, this reliability was evaluated by the methods of evidence analysis which influenced by various conducted techniques mong these methods, several studies assessed and evaluated the accuracy of bite mark analysis by manual docking and computer assisted overlay generation techniques.

On M. Jonathan and Ambiga Pazhani study, three food materials (apple, cheese and chocolate) were used to conduct bite marks and analyzed by computer assisted overlay generation technique and manual docking technique. They assessed the accuracy of the two techniques on the food materials and chocolate was found to be the most accurate food of the three for bite mark analysis. They compared the two methods by Spearman's correlation test, and they found that the manual docking technique was as accurate as the computer assisted overlay generation technique. (11)

In the current study, the bite marks were generated on three different food materials, Apple, chocolate and eggplant. We assessed the reliability of manual docking technique and computer aided overlay technique for analysis of the bite marks and all the three food substances were reliable for bite mark analysis with greater reproducibility on chocolate than eggplant and apple, as well the comparison between the two techniques was done by Spearman's correlation test and the results 
clarified the equality of the two techniques in accuracy for the bite mark analysis.

C. Stavrianos, et al. used the docking and the computer assisted overlay generation techniques to analyze a bite mark on an apple seized from the crime scene of their fabricated crime scenario in order to evaluate the accuracy of the later presenting two techniques on foodstuffs. The results showed that the two methods are similar in accuracy. Through the procedures of method application, the apple and the cast models of ten suspects participated as perpetrators were scanned electronically and the photos produced were used in the overlay production. At the end only one suspect's cast had an accurate matching to the apple's bite mark in both techniques. (12)

In the present study, each individual bite three food materials and the photos were produced by conventional $13 \mathrm{mbx}$ camera. The obtained results are dependent on non-parametric tests; Kruskal-Wallis $\mathrm{H}$ test to detect the accuracy among 45 bites' samples and Spearman's correlation test to compare between the docking and the computer assisted overlay generation technique.

\section{CONCLUSION}

According to the results of this study, the computer assisted overly generation is as accurate as the manual docking technique for bite mark analysis on food materials. Furthermore, the three food materials; the apple, the eggplant and the chocolate are reliable for bite mark analysis with the chocolate being the most accurate one.

\section{REFERENCES}

[1] Maloth S, Ganapathy KS. Comparison between five commonly used two-dimensional methods of human bite mark overlay production from the dental study casts. Indian Journal of Dental Research. 2011 May 1;22(3):493
[2] Vale GL, Noguchi TT. Anatomical distribution of human bite marks in a series of 67 cases. Journal of Forensic Science. 1983 Jan 1;28(1): 61-9.

[3] Verma K, Joshi B, Joshi CH, Paul MP. Bite marks as physical evidence from the crime scene-an overview. J Scientific Reports. 2013; 2:605-10.

[4] Stavrianos C, Vasiliadis L, Papadopoulos C, Pantazis A, Petalotis N, Tatsis D, Samara E. A case report of facial bite marks; reference of methods of analysis. Res J Med Sci. 2011;5(3):126-32.

[5] Bowers CM, Johansen RJ. Digital rectification and resizing correction of photographic bite mark evidence. Forensic Science Communications. 2001 Jul 1;3(3).

[6] Kouble RF, Craig GT. A survey of the incidence of missing anterior teeth: potential value in bite mark analysis. Science \& Justice. 2007 May 31;47(1):19-23.

[7] Van der Velden A, Spiessens M, Willems G. Bite mark analysis and comparison using image perception technology. Journal of Forensic Odontostomatology. 2006 Jun 1;24(1):14.

[8] Tai MW, Chong ZF, Asif MK, Rahmat RA, Nambiar P. A comparative study between xerographic, computer-assisted overlay generation and animated-superimposition methods in bite mark analyses. Legal Medicine. 2016 Sep 30; 22:42-8.

[9] Bhargava K, Bhargava D, Rastogi P, Paul M, Paul R, Jagadeesh HG, Singla A. An overview of Bite mark analysis. Pretty IA. Forensic dentistry: 2. Bitemarks and bite injuries. Dental Update-London .2008;35(1):48.

[10] Daniel MJ, Pazhani A. Accuracy of bite mark analysis from food substances: A comparative study. Journal of forensic dental sciences. 2015 Sep;7(3):222.

[11] Stavrianos C, Vasiliadis L, Emmanouil J, Papadopoulos C. In vivo evaluation of the accuracy of two methods for the Bitemark analysis in food stuff. Res J Med Sci. 2011; 5(1):25-31.

Citation: Nima A. Osman, Aya Z. Omer and Amal H. Abuaffan. Comparative Study on Two Methods for Bite Mark Analysis. ARC Journal of Forensic Science. 2017 2(1):12-16. http://dx.doi.org/10.20431/24560049.0201004

Copyright: (C) 2017 Authors. This is an open-access article distributed under the terms of the Creative Commons Attribution License, which permits unrestricted use, distribution, and reproduction in any medium, provided the original author and source are credited. 